\title{
La réserve électronique aux Bibliothèques de l'Université Concordia
}

\section{Introduction}

Avec une croissance de plus de $10 \%$ de son corps étudiant depuis cinq ans, l'Université Concordia s'acharne à répondre aux besoins diversifiés de ses 30000 étudiants à temps plein et partiel. Sa bibliothèque dispose de deux points de service, sur chacun des campus universitaires à Montréal au Canada. La communauté étudiante dispose déjà de plusieurs services virtuels, dont un Catalogue interrogeable en ligne permettant le renouvellement des prêts, des services de référence par courriel (email) et clavardage (chat) en plus d'une multitude de bases de données en accès commuté.

En effet, la bibliothèque mise fortement sur les opportunités du monde digital pour bonifier son offre de service. En janvier 2004, la bibliothèque se lança dans un ambitieux projet, celui d'incorporer un nouveau module à son catalogue en ligne. Ce dernier permet à la bibliothèque de verser des documents électroniques dans son catalogue web, puis aux usagers de les télécharger à partir de n'importe quel ordinateur connecté à Internet. La bibliothèque espère que ce module de gestion de contenu multimédia facilitera grandement le processus de réserve des professeurs d'ici la rentrée en septembre 2004.

La réserve est un service bien répandu en Amérique du Nord. Sommairement, les professeurs consignent des documents « papier » à la bibliothèque pour appuyer leur enseignement. Ces documents sont des notes de cours, des exercices et leurs solutionnaires, des examens antérieurs, des photocopies d'articles académiques ou de chapitres de livres, voire même des livres entiers qui sont consultés par les étudiants lors de la session. Malgré la simplicité de ce service, de longues files d'attente guettent les étudiants à la mi- et la fin session pour l'emprunt et la photocopie des documents, plusieurs pages sont égarées ou rendues illisibles par l'usure, sans oublier la nécessité de se conformer aux heures 
d'ouverture de la bibliothèque. La réserve électronique vise à contrecarrer ces problèmes d'accès.

Comme dans tout projet technologique, la bibliothèque doit faire face à plusieurs choix pour l'implantation de ce nouveau module à son catalogue. Comment modifier le service «papier » pour incorporer l'offre de service électronique ? Est-il pertinent de numériser toutes les demandes « papier»? Devons-nous imposer une limite à la taille des fichiers ? Et que faire des droits d'auteurs ? Malheureusement, il est difficile de se référer aux autres bibliothèques canadiennes puisque seulement quatre des trente membres de l'Association des bibliothèques de recherche du Canada offrent un service similaire, et aucun au Québec. La bibliothèque a créé un groupe de travail, constitué de trois bibliothécaires, un responsable de service et un commis senior, pour piloter l'implantation. Comme nous allons voir, les problématiques institutionnelles se sont révélées plus importantes que les considérations technologiques.

Afin de présenter l'implantation du service des réserves électroniques d'une manière systématique et cohérente, nous allons employer une méthodologie d'analyse de cas technologiques nommée le « Cadre d'analyse centré sur la tâche » (Work Centered Analysis Method) de Steven Alter (1999). En fait, le but de cet exposé est double : vous présenter cette méthodologie d'analyse tout en exposant le cas précis en question. Vous pourrez donc comprendre ce qui ressort de la situation à la bibliothèque de l'Université Concordia, tout en retenant un gabarit d'analyse que vous pourrez appliquer dans votre contexte institutionnel. Nous terminerons en tirant des leçons de notre implantation.

\section{Développement}

\section{Présentation du Cadre d'analyse centré sur la tâche}

Avant tout, pourquoi utilisons-nous un cadre d'analyse (ou méthodologie d'analyse)? Comme disait Descartes dans son Discours de la méthode, c'est pour échapper 
au hasard et éviter de se perdre ! Dit autrement, c'est simplement pour organiser, d'une façon globale, cohérente et succincte, les problématiques décisionnelles liées à un cas de gestion de systèmes d'information. Les détails et les considérations deviennent rapidement insurmontables si une approche systématique n'est pas retenue pour structurer l'approche. Par ailleurs, un cadre d'analyse permet d'agir comme aide-mémoire afin de couvrir tous les aspects de l'implantation technologique. La sagacité cartésienne s'applique toujours au monde d'Internet!

Il existe plusieurs cadres d'analyse pour étudier un système d'information, mais celui retenu fut le Cadre d'analyse centré sur la tâche de Steven Alter (Alter, 1999). Sa simplicité et sa rigueur en sont ses deux points forts. Pour en savoir plus sur les méthodologies d'analyse de systèmes d'information, veuillez consulter un manuel couvrant l'analyse et la modélisation de systèmes d'information.

Le Cadre d'analyse centré sur la tâche se présente comme une pyramide où chaque élément vient s'insérer comme une pièce d'un puzzle. À la tête de celui-ci se trouve les clients du système d'information. Il s'agit d'agents ou de personnes externes qui ont avantage à interagir avec le mécanisme pour en obtenir un produit d'information. Immédiatement sous les clients se trouvent les produits du système, la raison pour laquelle les clients sont là. Viennent ensuite les processus d'affaires, qui créent les produits d'information. Les processus d'affaires sont appuyés par les participants internes au système, par l'information requise pour faire fonctionner le système, puis par la technologie. La figure 1 donne un aperçu graphique du Cadre d'analyse centré sur la tâche. 


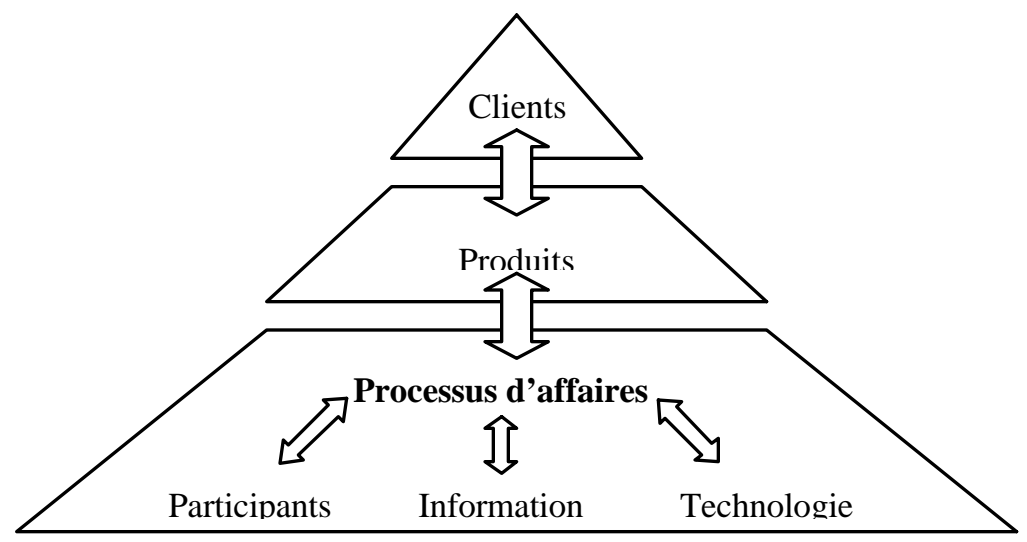

Figure 1 Cadre d'analyse centré sur la tâche (Alter 1999)

Constatez que les processus d'affaires se retrouvent au centre de la pyramide. Vous pouvez donc comprendre l'origine du nom de ce cadre d'analyse : la tâche est en effet le processus d'affaires qui crée les produits d'information recherchés par les clients. Ils se basent sur les participants internes, l'information et la technologie pour exister. Par ailleurs, remarquez que ce cadre d'analyse est très conceptuel et permet d'appréhender un système complexe en quelques rubriques simples et mutuellement exclusives. Généralement, nous appuyons une telle représentation de haut niveau par d'autres outils d'analyse, comme des schémas de procédés, de flux de données et des dictionnaires de données. Ceci n'est que la pointe de l'iceberg de l'analyse conceptuelle des systèmes et un bon manuel permettra de compléter le portrait.

Normalement, nous appliquons ce cadre d'analyse en deux temps, c'est-à-dire avant le changement technologique, puis après le changement. Deux Cadres d'analyse centrés sur la tâche sont donc créés. Cette approche permet d'analyser les modifications au système et fait ressortir clairement l'évolution de chacune des pièces du puzzle. Nous devons malheureusement limiter l'exposé au contexte final, afin de respecter les délais et le thème de l'exposé. Passons maintenant à l'application du Cadre d'analyse centré sur la tâche au cas de la réserve électronique des Bibliothèques de l'Université Concordia. 


\section{Application du cadre d'analyse}

\section{Facteurs externes}

\section{Clients - utilisateurs finaux du système}

Il est simple d'identifier les étudiantes et étudiants de l'Université comme les utilisateurs finaux du système. En effet, les 20000 universitaires à temps plein et les 10000 à temps partiel doivent faire appel au catalogue afin de repérer les documents versés à la réserve par les professeurs. Par ailleurs, un système d'authentification bloque l'accès aux autres internautes.

\section{Produits - l'offre, les extrants du système}

Sommairement, les documents consignés à la réserve par les professeurs sont les produits ciblés de ce système. Malheureusement, le Groupe de travail s'est rapidement heurté à un mur dans l'élaboration de l'offre de service de numérisation. Outre les considérations technologiques (qui seront discutées dans quelques instants), une attention particulière fut portée à la problématique du droit d'auteur lors de la rediffusion d'ouvrages nouvellement numérisés. La cadre réglementaire canadien applicable ressemble à un oignon : plusieurs couches successives entrent en jeu et une dissection de la chose nous donne rapidement le goût de pleurer. En plus de la loi proprement dite, nous avons consulté la politique universitaire (disponible en ligne), plusieurs de nos contrats de licence de bases de données «plein texte », la volumineuse documentation du processus de réforme du droit d'auteur canadien présentement en cours ainsi que quelques jugements de la cour, sans oublier le bureau du contentieux. Donc, quels ouvrages publiés pouvons-nous numériser? La réponse, en quatre mots, est : nous ne savons pas!

Mais tout n'est pas perdu. Le Groupe de travail, dans un souci de respecter les délais d'implantation et la loi, a opté pour la numérisation des documents créés par les professeurs. En effet, ces derniers contrôlent toujours les droits de distribution de leurs 
notes de cours, examens et exercices. Les bibliothèques peut donc facilement obtenir leur consentement pour la numérisation et la diffusion en ligne de ces documents. Aussi, nous avons identifié les sites Internet et les articles accessibles grâce à nos bases de données «plein texte » comme autres sources à cibler. Ces documents sont déjà numérisés et nous pouvons facilement créer un hyperlien dans notre catalogue sans heurter le droit des créateurs.

Pour tout dire, le flou législatif a poussé le Groupe de travail à limiter l'offre de service de numérisation et d'accès en ligne aux documents créés par nos enseignants, aux sites Internet et aux articles «plein texte » de nos bases de données. Sont donc exclus tous les livres et photocopies d'articles, ce qui représente environ les deux tiers des documents à la réserve.

\section{Facteurs internes}

\section{Processus d'affaires - comment procéder, les étapes du système}

Il y a trois processus principaux rattachés à ce système : le processus d'ajout de documents à la réserve électronique, le processus de désherbage de la réserve électronique ainsi que le processus de repérage de documents par les étudiants. Ce dernier ne varie que légèrement puisque l'usager doit simplement cliquer sur le titre du document dans le catalogue en ligne pour accéder à la notice où se trouve l'hyperlien pour le téléchargement (au lien de noter la cote du document et se présenter au comptoir de circulation). Par contre, les deux autres processus demandent plus d'attention. Examinons-les rapidement.

Le processus d'ajout de documents à la réserve électronique varie en fonction de l'origine de la demande du professeur. Si la demande provient du processus de réserve régulier, c'est-à-dire que le professeur télécharge le formulaire de la réserve du site Internet de la bibliothèque et achemine sa demande via courrier interne ou en personne, le commis du comptoir de la circulation examine chaque document de la demande. Pour chaque article 
de périodique demandé, ce dernier vérifie si une copie numérique est accessible par le truchement de nos bases de données en ligne et, dans tel cas, crée un hyperlien à l'article en ligne sur la notice du document (sinon, nous n'avons que la copie papier soumise par le professeur). Aussi, pour chaque document créé par le professeur (notes de cours, exercices, etc.), le commis procède à la numérisation et le document « Acrobat Reader » ou «PDF » est ainsi consigné dans notre catalogue. Nous ne numérisons que si le professeur a coché une case spéciale du formulaire de demande de réserve et a dûment signé la demande. Veuillez noter que, dans ces deux cas, la copie papier ainsi que la copie numérique sont conservées par les bibliothèques.

Une alternative qui s'offre au professeur est d'utiliser un formulaire dynamique en ligne pour automatiquement envoyer son document en format électronique à la bibliothèque. Notre serveur Internet envoie automatiquement un courriel à l'équipe du bureau de circulation pour les informer de la présence du document sur nos serveurs. Notre équipe peut donc créer la notice dans le catalogue pour ce document électronique. Cette méthode a l'avantage de se faire totalement sur Internet.

Pour ce qui est du désherbage, l'équipe du comptoir de circulation doit simplement s'assurer de supprimer les fichiers électroniques du serveur Internet assigné à la réserve électronique lorsque les cours sont terminés et que les réserves sont purgées.

\section{Participants - ressources ou agents internes affectés au système}

Les agents internes au système de la réserve électronique sont les suivants :

- Les professeurs puisqu'ils initient le processus de la réserve électronique et fournissent l'information requise;

- L'équipe du comptoir de la circulation des deux bibliothèques puisqu'elle assure la numérisation et manipule les fichiers;

- Le groupe de travail sur les réserves électroniques (trois bibliothécaires, un responsable de département, un commis sénior) par leur conception des politiques et procédures du service; 
- L'équipe technique de la bibliothèque pour la configuration et le soutien des différents serveurs Internet.

Constatez qu'il est particulièrement utile d'identifier les participants au système pour assurer la formation adéquate.

\section{Information -données nécessaires au système}

Nous avons besoin d'information à propos du professeur, de son cours ainsi que certains éléments concernant les documents eux-mêmes pour faire fonctionner le système adéquatement. Avant tout, l'accès principal aux documents dans le catalogue s'opère soit par le sigle de cours, soit par le nom de famille du professeur. Nous demandons également certaines données afin de retracer les professeurs en cas de problèmes ou simplement pour retourner leurs copies personnelles de livres. Les photocopies sont habituellement recyclées.

Pour ce qui est des documents personnels comme des notes de cours, nous devons savoir quel sera leurs titres dans le catalogue. Aussi, certaines données concernant la manipulation des documents sont requises, comme la permission de numériser et la durée du cours (une session, un an). Finalement, les professeurs doivent fournir une référence bibliographique complète pour les articles de périodiques afin de pouvoir les retracer dans nos bases de données.

\section{Technologie}

Ce dernier point - mais non le moindre! - comprend les éléments suivants :

- Le module de gestion de contenu multimédia (Media Management Module) de de notre catalogue web Innovative Interfaces (notez que ce module peut gérer ces documents MS Word, RTF, TXT, HTML, PDF, PPT, ainsi que la plupart des format d'images);

- Formulaires et serveurs Internet pour verser les documents en ligne qui utilisent la technologie PHP, CGI et HTTP pour héberger les fichiers numériques;

- Numérisateur à balayage (ou «scanneur») pour les documents papier; 
- Navigateur Web pour extraire l'information par les usagers.

Tous ces éléments techniques entrent en ligne de compte pour le bon fonctionnement de la réserve électronique.

\section{Conclusion}

Grâce au Cadre d'analyse centré sur la tâche, nous avons pu, simplement et rapidement, brosser un portrait complet de ce nouveau service en ligne. Les réserves électroniques, disponibles à travers le catalogue en ligne vont permettre aux Bibliothèques de l'Université Concordia de bonifier ce point de contact avec l'usager afin de leur offrir un nouveau service virtuel.

En guise de conclusion, voici quelques leçons qui ont fait surface lors de l'implantation de ce nouveau service en ligne :

- Il est souvent plus facile d'implanter un projet pilote pour apprendre de nos erreurs et s'attaquer à une situation plus simplifiée;

- Le cadre technologique est assurément influencé par le cadre législatif et réglementaire (droit d'auteur);

- Les participants internes au système sont tout aussi importants (sinon plus) que la technologie! (formation de l'usager, résistance au changement);

- Il faut toujours se positionner par rapport aux produits ou services qui entrent en compétition avec le nouveau processus, par exemple les sites Internet personnels des professeurs.

En se tournant vers l'avenir, ce service pourrait être étendu à d'autres types de documents comme les thèses de nos étudiantes et étudiants aux cycles supérieurs, voire même aux articles pré-publiés de nos chercheurs. Une fois le système en marche, il ne faut que garder l'oreille tendue pour identifier de nouvelles synergies et opportunités. Merci de généreuse attention! 


\section{Bibliographie}

ALTER, Steven (1999) Information Systems: a Management Perspective. Upper Saddle River, NJ : Prentice-Hall.

Soros Foundation. Budapest Open Access Initiative. Page accessed on 2003-12-12. URL: http://www.soros.org/openaccess/

Prentice-Hall. Information Systems - The Work Centered Analysis Method. Page accessed on 2003-12-12. URL:

http://www.prenhall.com/divisions/bp/app/alter/about/wca.html 